\title{
A!
}

This is an electronic reprint of the original article.

This reprint may differ from the original in pagination and typographic detail.

Caicedo-Dávila, S.; Lopez-Acevedo, O.; Velasco-Medina, J.; Avila, A.

Density and localized states' impact on amorphous carbon electron transport mechanisms

Published in:

Journal of Applied Physics

DOI:

$10.1063 / 1.4971010$

Published: 07/12/2016

Document Version

Publisher's PDF, also known as Version of record

Please cite the original version:

Caicedo-Dávila, S., Lopez-Acevedo, O., Velasco-Medina, J., \& Avila, A. (2016). Density and localized states' impact on amorphous carbon electron transport mechanisms. Journal of Applied Physics, 120(21), 1-9. [214303]. https://doi.org/10.1063/1.4971010

This material is protected by copyright and other intellectual property rights, and duplication or sale of all or part of any of the repository collections is not permitted, except that material may be duplicated by you for your research use or educational purposes in electronic or print form. You must obtain permission for any other use. Electronic or print copies may not be offered, whether for sale or otherwise to anyone who is not an authorised user. 


\section{Density and localized states' impact on amorphous carbon electron transport mechanisms}

S. Caicedo-Dávila, O. Lopez-Acevedo, J. Velasco-Medina, and A. Avila

Citation: Journal of Applied Physics 120, 214303 (2016); doi: 10.1063/1.4971010

View online: https://doi.org/10.1063/1.4971010

View Table of Contents: http://aip.scitation.org/toc/jap/120/21

Published by the American Institute of Physics

\section{Articles you may be interested in}

Accurate schemes for calculation of thermodynamic properties of liquid mixtures from molecular dynamics simulations

The Journal of Chemical Physics 145, 244504 (2016); 10.1063/1.4973001

Appropriate use of the particle-in-cell method in low temperature plasmas: Application to the simulation of negative ion extraction

Journal of Applied Physics 120, 213303 (2016); 10.1063/1.4971265

Influence of $90^{\circ}$ charged domain walls on the electrocaloric effect in $\mathrm{PbTiO}_{3}$ ferroelectric thin films

Journal of Applied Physics 120, 214105 (2016); 10.1063/1.4971400

Modeling of reduced effective secondary electron emission yield from a velvet surface

Journal of Applied Physics 120, 213302 (2016); 10.1063/1.4971337

Smart viscoelastic and self-healing characteristics of graphene nano-gels

Journal of Applied Physics 120, 214304 (2016); 10.1063/1.4971267

Structural transition and its effect in $\mathrm{La}, \mathrm{Zr}$ co-substituted mono-domain $\mathrm{BiFeO}_{3}$

Journal of Applied Physics 120, 214106 (2016); 10.1063/1.4969047

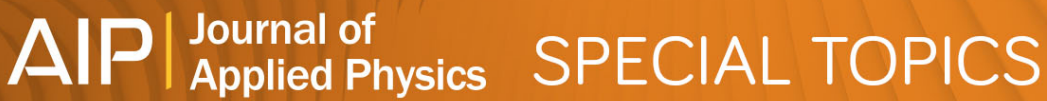

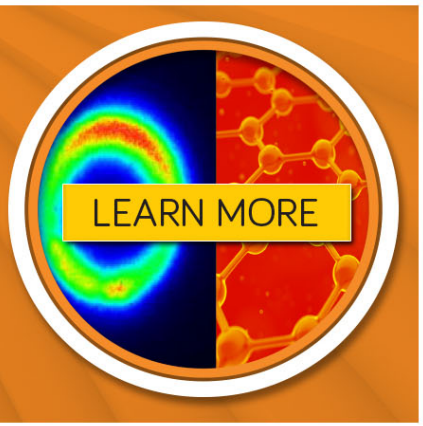




\title{
Density and localized states' impact on amorphous carbon electron transport mechanisms
}

\author{
S. Caicedo-Dávila, ${ }^{1, \text { a) }}$ O. Lopez-Acevedo, ${ }^{2}$ J. Velasco-Medina,${ }^{3}$ and A. Avila ${ }^{1}$ \\ ${ }^{1}$ Department of Electrical and Electronic Engineering and Centro de Microelectrónica (CMUA), \\ Universidad de los Andes, Bogotá, Colombia \\ ${ }^{2}$ COMP Centre of Excellence in Computational Nanoscience, Department of Applied Physics, \\ Aalto University, Espoo, Finland \\ ${ }^{3}$ School of Electrical and Electronic Engineering, Universidad del Valle, Cali, Colombia
}

(Received 24 August 2016; accepted 16 November 2016; published online 5 December 2016)

\begin{abstract}
This work discusses the electron transport mechanisms that we obtained as a function of the density of amorphous carbon (a-C) ultra-thin films. We calculated the density of states (total and projected), degree of electronic states' localization, and transmission function using the density functional theory and nonequilibrium Green's functions method. We generated 25 sample a-C structures using ab-initio molecular dynamics within the isothermal-isobaric ensemble. We identified three transport regimes as a function of the density, varying from semimetallic in low-density samples $\left(\leq 2.4 \mathrm{~g} / \mathrm{cm}^{3}\right)$ to thermally activated in high-density $\left(\geq 2.9 \mathrm{~g} / \mathrm{cm}^{3}\right)$ tetrahedral a-C. The middle-range densities $\left(2.4 \mathrm{~g} / \mathrm{cm}^{3} \leq \rho \leq 2.9 \mathrm{~g} / \mathrm{cm}^{3}\right)$ are characterized by resonant tunneling and hopping transport. Our findings offer a different perspective from the tight-binding model proposed by Katkov and Bhattacharyya [J. Appl. Phys. 113, 183712 (2013)], and agree with experimental observations in low-dimensional carbon systems [see S. Bhattacharyya, Appl. Phys. Lett. 91, 21 (2007)]. Identifying transport regimes is crucial to the process of understanding and applying a-C thin film in electronic devices and electrode coating in biosensors. Published by AIP Publishing.

[http://dx.doi.org/10.1063/1.4971010]
\end{abstract}

\section{INTRODUCTION}

Amorphous carbon (a-C) is a noncrystalline solid allotropic form of carbon with a combination of $s p^{2}$ and $s p^{3}$ hybridized atoms. The ratio of bonds determines its density and different material classes and properties: from lowdensity $s p^{2}$-rich non-graphitizing a-C, to high-density $s p^{3}$ rich tetrahedral a-C (ta-C). The a-C thin films' electrical properties make them attractive for electronic devices and as electrode coating in electrochemical biosensors, ${ }^{1-5}$ as shown by their inclusion in the Emerging Research Devices chapter of the 2014 report, International Technology Roadmap for Semiconductors. ${ }^{6}$ For this reason, understanding and predicting electron transport mechanisms and electrical properties is vital to advance a-C-based technology.

Bhattacharyya ${ }^{7}$ explained different transport mechanisms from experimental measurements of a-C, correlating the conduction with the density of states (DOS) at the Fermi level $\left(E_{F}\right)$. Some recent models ${ }^{8-10}$ attempted to describe the transport phenomena of $\mathrm{a}-\mathrm{C}$ at the tight-binding level of theory, considering regions of concentrated $s p^{3}$ and $s p^{2}$ atoms as barriers and wells. These models need further refinement to include the random nature of the material's atomic structure, low-dimensionality, and coupling with electrodes in very thin films, because they affect the transport mechanisms in lowdimensional structures, as observed experimentally. ${ }^{1,11,12}$

Although atomistic simulations have been used to model $\mathrm{a}-\mathrm{C}$, the lack of long-range order limits the system size

${ }^{a)}$ Electronic mail: s.caicedo14@uniandes.edu.co possible to simulate. Parametric and/or semi-empirical approaches have been proposed to overcome this limitation, ${ }^{13}$ but at the expense of calculated properties' accuracy. Atomistic ab-initio simulations based on density functional theory (DFT) remain the most accurate and affordable technique to calculate a-C properties from the electronic structure, for small- to medium-size systems. ${ }^{14,15}$ These simulations have focused on computational generation strategies, analysis of the atomic structure, and optical and mechanical properties. ${ }^{14-20}$ Combining DFT and nonequilibrium Green's functions (DFT-NEGF) has proven useful for qualitatively describing and investigating electron transport in nano and molecular systems, materials interfaces, and functional electronic devices and sensors. ${ }^{21-24}$ Despite the importance of the theoretical models established for electrical and transport properties, ${ }^{25}$ to our knowledge, a-C transport properties have not been studied from first-principles calculations. Thus, here, we used DFT-NEGF to investigate the electron transport mechanisms in ultra-small a-C structures, analyzing the effects of density, dimensionality, and contact with metallic electrodes. We were able to explain different types of conductions observed experimentally: ${ }^{7,26,27}$ semimetallic in lowdensity a-C films, and thermally activated for $s p^{3}$ rich a-C. Our calculations also support experimental evidence of resonant tunneling (RT) in ultra-thin films, ${ }^{1,11,12}$ and establish three regimes of dominant mechanisms, as a function of the density.

Recent work ${ }^{19}$ demonstrated that the compressive pressure is an important variable to generate a-C structures. In their work, the authors obtained the desired pressure by 
readjusting the cell size followed by relaxation until reaching the target conditions. We propose a generalization of this generation strategy using Born-Oppenheimer Molecular Dynamics (MD) within the isothermal-isobaric ensemble (NPT). With this generation strategy, we can increase the sampling size for statistical purposes.

The computational results presented here are important in the context of predictive modeling and engineering of materials with specific electrical properties, especially when considering the materials' aimed industrial applications.

\section{COMPUTATIONAL DETAILS AND METHODS}

We carried out our calculations with the Kohn-Sham DFT method, ${ }^{28}$ using the Perdew-Burke-Ernzerhof (PBE) generalized gradient approximation (GGA) for the exchangecorrelation functional, ${ }^{29}$ as implemented in the GPAW code. ${ }^{30}$ GPAW is a grid-based implementation of the projectoraugmented wave (PAW) method. ${ }^{31}$ Unless otherwise specified, the calculations used a plain waves (PW) basis set with the cutoff set to $400 \mathrm{eV}$ for the a-C generation and a double- $\zeta$ polarized (DZP) linear combination of atomic orbitals (LCAO) basis set for the transport calculations, which yields good convergence for a-C systems. ${ }^{32,33}$ The $k$-space integration was done over a $(4 \times 4 \times 1)$ Monkhorst-Pack grid. ${ }^{34}$ The atomic positions were optimized following the Broyden-FletcherGoldfarb-Shanno (BFGS) algorithm ${ }^{35-38}$ until all the forces acting on the atoms were below $0.05 \mathrm{eV} / \AA$.

\section{A. Bulk a-C generation method}

We built 25 orthogonal supercells of 64 randomly positioned carbon atoms. The dimensions of each supercell in the $x y$ plane are multiples of the diamond unit cell (111) plane $(5.8 \AA \times 5.0 \AA)$, and the $z$ component was adjusted so that the sum of the atoms' masses divided by the volume of the cell yielded a target density. After a full relaxation, the system was equilibrated at $300 \mathrm{~K}( \pm 10 \mathrm{~K})$ using the Berendsen algorithm $^{39}$ within the canonical ensemble (NVT dynamics). After equilibration at $300 \mathrm{~K}$, the temperature was kept constant using the Berendsen algorithm within the frame of isothermal-isobaric (NPT) dynamics, with a target compressive pressure, calculated from a linear fit of experimental data by Fallon et al. ${ }^{40}$

$$
P=0.0718 * f_{s p^{3}}+3 .
$$

Here $P$ is the target compressive pressure in GPa, and $f_{s p^{3}}$ is the system's $s p^{3}$ fraction, which was calculated from fitted experimental data by Ferrari et al. ${ }^{41}$

$$
f_{s p^{3}}=70.73 * \rho-132.91,
$$

where $\rho$ is the target density of the sample in $\mathrm{g} / \mathrm{cm}^{3}$.

The DOS and projected DOS (PDOS) of the sample used to analyze the electronic structure and determine a-C electronic properties are calculated by means of the converged Kohn-Sham eigenstates $\left|\psi_{n}\right\rangle$, with eigenvalue $\varepsilon_{n}$

$$
g(E)=\sum_{n}\left\langle\psi_{n} \mid \psi_{n}\right\rangle \delta\left(E-\varepsilon_{n}\right)
$$

$$
g_{\alpha, i}(E)=\sum_{n}\left|\left\langle\phi_{i}^{\alpha} \mid \psi_{n}\right\rangle\right|^{2} \delta\left(E-\varepsilon_{n}\right)
$$

Here $g(E)$ is the DOS, $E$ is the energy variable, $g_{\alpha, i}(E)$ is the PDOS, and $\left|\phi_{i}^{\alpha}\right\rangle$ is the $i^{\text {th }}$ atomic orbital of atomic site $\alpha$. To compensate for finite $k$-space sampling, the states are smeared with a Gaussian function centered at $\varepsilon_{n}\left[\delta\left(E-\varepsilon_{n}\right)\right.$ in Equations (3) and (4)]. The width of the Gaussian function was set to $0.01 \mathrm{eV}$ to better show the features in the electronic density of states. Note that the width of the Gaussian is smaller than the electronic smearing set to $0.1 \mathrm{eV}$ in the calculations.

We defined the hybridization of an atom by counting the number of neighbors within a cutoff radius of $1.9 \AA$, considered the bonding distance: an $s p^{2}$ atom has three neighbors, while an $s p^{3}$ atom has four neighbors. This definition and cutoff radius have been used in the literature, obtained from the analysis of the radial distribution function. ${ }^{15-17,19,33,42}$ After labeling each atomic site, it is easy to qualitatively assess the contributions of $s p^{2}$ and $s p^{3}$ sites to the DOS by summing the contributions of all the sites in the system

$$
g_{s p^{2} / s p^{3}}(E)=\sum_{\alpha=s p^{2} / s p^{3}} \sum_{i=2 s, 2 p} g_{\alpha, i}(E),
$$

where $g_{s p^{2} / s p^{3}}(E)$ is the contribution of $s p^{2}$ or $s p^{3}$ sites to the DOS.

Finally, we define the local DOS (LDOS) as the sum of $g_{\alpha, i}$ over the valence states $i$ of all the sites $\alpha$ in a particular region in space.

\section{B. Transport system}

The generated a-C samples were used to build a system for electron transport calculations that consists of a scattering region and left and right electrodes. The scattering region was built with a molecule (a-C sample) and two principal layers (PL), which in our case are slabs of $3 \mathrm{fcc}$ (111)Au atomic layers (a more extensive discussion on PL is found elsewhere ${ }^{43-47}$ ). The $\mathrm{Au} / \mathrm{a}-\mathrm{C}$ interfaces in the scattering region were relaxed to prevent abrupt changes in the electrostatic potential. The interface was modeled with a reduced system consisting of an a-C slab, and built taking the atoms within a $5 \AA$ surface measured from the topmost $\mathrm{C}$ atom. For the bottom atoms a similar slab was defined. The surface was passivated with hydrogen to compensate for dangling bonds. ${ }^{19}$ This slab and the PL were relaxed at six different distances from each other, between 1.5 and $3.5 \AA$, in a unit cell adding $10 \AA$ vacuum up and down in the $z$ direction to avoid orbital overlapping and periodicity effects. The atoms in the outermost $2 \AA$ surface of the a-C slab and last atomic layer of the PL were fixed, so that the transport system could be reconstructed easily. Boundary conditions can cause an electrostatic potential gradient in the vacuum region (see the blue line in Fig. 1). The gradient creates an artificial electric field in the system, which may lead to nonphysical effects during the relaxation. ${ }^{48}$ To ensure vacuum neutrality we set periodic boundary conditions in the $x$ and $y$ directions, and fixed boundary conditions with dipole correction in the $z$ direction (see the red line in Fig. 1). 

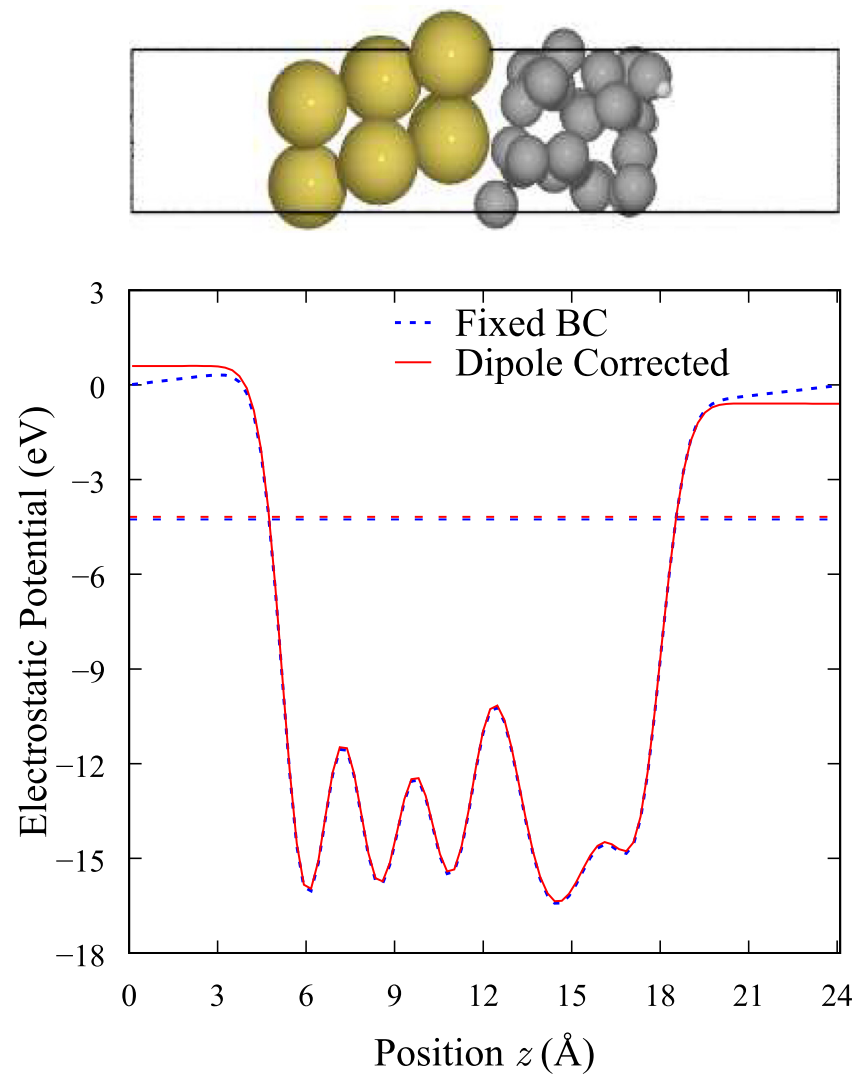

FIG. 1. The top panel shows the fully relaxed atomic structure at the Au/a-C interface. Yellow, gray, and white spheres represent $\mathrm{Au}, \mathrm{C}$, and $\mathrm{H}$ atoms, respectively. The bottom panel shows the electrostatic potential with (see the red solid line) and without (blue dotted line) dipole correction of a $3.2 \mathrm{~g} /$ $\mathrm{cm}^{3}$ sample. The Fermi level is shown as a dashed horizontal line.

The total energy of the six relaxed interfaces as a function of the $\mathrm{Au} / \mathrm{a}-\mathrm{C}$ distance was fixed to a quadratic polynomial. The minimum is assumed to be the optimum interface distance, which lies between 2.5 and $3 \AA$ for all the generated samples, as Fig. 2 shows. The interfaces are finally relaxed using the calculated optimum distance shown in the top

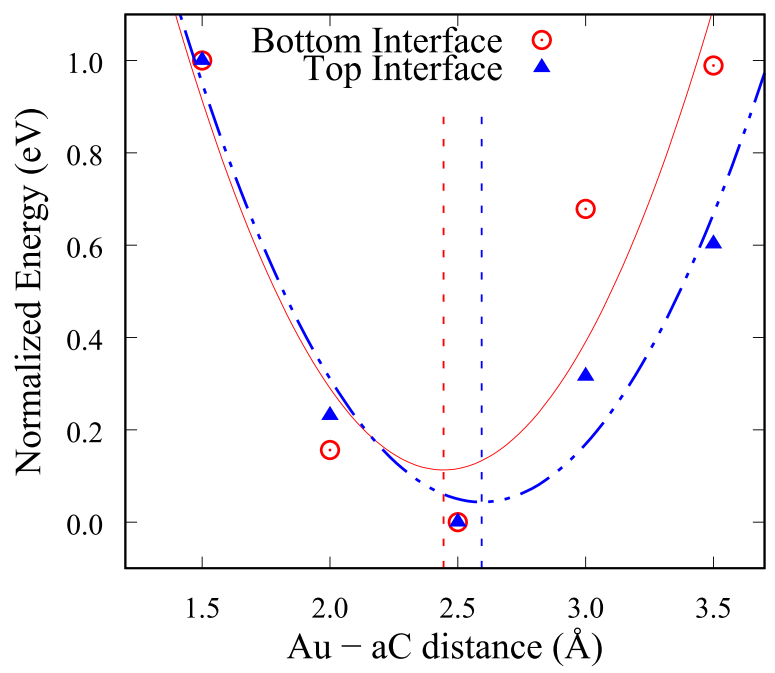

FIG. 2. Normalized energy of the relaxed interfaces, calculated as a function of the $\mathrm{Au} / \mathrm{a}-\mathrm{C}$ distance for a $3.2 \mathrm{~g} / \mathrm{cm}^{3}$ sample. We show the fixed quadratic curves and the equilibrium distance (dashed vertical lines). panel of Fig. 1, and the scattering region is reconstructed and connected to the electrodes shown in Figs. 3(a) and 3(b).

The transport properties were calculated by means of the retarded Green's function, ${ }^{49,50}$ as implemented in the GPAW code $^{47}$

$$
G^{r}(E)=\left[E S-H-\Sigma^{r}(E)\right]^{-1},
$$

where $E$ is the energy variable, $\Sigma^{r}(E)=\sum_{i} \Sigma_{i}^{r}(E)$ is the sum of self-energies $\Sigma_{i}^{r}(E)$ (one for each electrode, in our case), and $S$ and $H$ are the overlap and Hamiltonian matrices of the scattering region, respectively (calculated self-consistently from the nonequilibrium electron density as detailed elsewhere ${ }^{47}$ ). Using Equation (6), we can calculate the Transmission function as follows:

$$
T(E)=\operatorname{Tr}\left[\Gamma_{1}^{r}(E) G^{r}(E) \times \Gamma_{2}^{r}(E) G^{r}(E)^{\dagger}\right],
$$

where $\Gamma_{n}^{r}(E)=i\left(\Sigma_{n}^{r}(E)-\Sigma_{n}^{r}(E)^{\dagger}\right)$ is the broadening matrix, the antihermitian part of the individual self-energies for each electrode. We can calculate the current as a function of energy, and therefore applied bias, by integrating Equation (7) in the Landauer Formula ${ }^{49}$

$$
I=\frac{q}{h} \int T(E)\left(f_{1}(E)-f_{2}(E)\right) d E,
$$

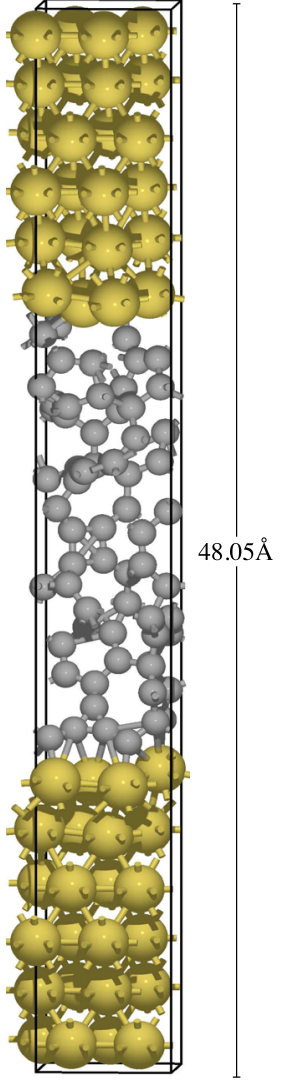

(a)

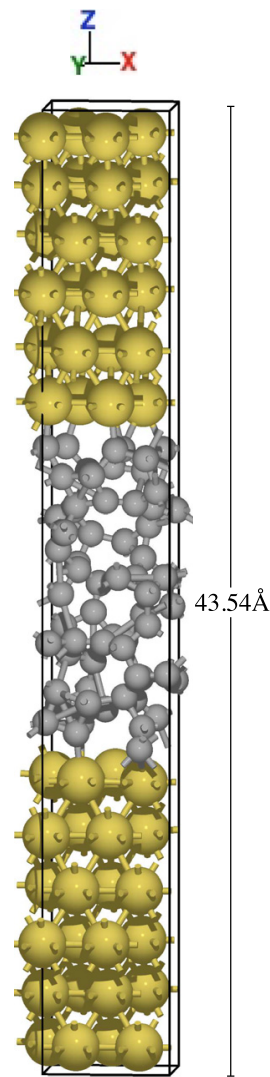

(b)
FIG. 3. (a) Atomic structure of low-density $\left(2.29 \mathrm{~g} / \mathrm{cm}^{3}\right)$ and (b) highdensity $\left(3.20 \mathrm{~g} / \mathrm{cm}^{3}\right)$ systems used in quantum transport calculations. Yellow and gray spheres represent $\mathrm{Au}$ and $\mathrm{C}$ atoms, respectively. 
where $q$ is the electron charge, $h$ the Planck's constant, and $f_{n}(E)=f\left(E-\mu_{n}\right)$ is the Fermi distribution function of electrode $n$. We used an electron smearing of $k T=0.1 \mathrm{eV}$ and $\mu_{n}=E_{F} \pm q V / 2$, with $E_{F}$ being the equilibrium Fermi level of the Au electrodes, and $V$ the applied voltage.

\section{RESULTS AND DISCUSSION}

\section{A. Bulk a-C generation}

Fig. 4(a) shows the results of $\rho$ versus $s p^{3}$ for the generated a-C structures. The NPT method yields structures closer to the experiment, ${ }^{41}$ compared with other theoretical approaches, such as Carr-Parrinello molecular dynamics (CPMD) by Marks et al. ${ }^{17}$ The slope fitted for CPMD is $37.53\left(\mathrm{~g} / \mathrm{cm}^{3}\right)^{-1}$, while for our NPT calculation it is 58.51 $\left(\mathrm{g} / \mathrm{cm}^{3}\right)^{-1}$. The slope fitted from experimental data ${ }^{41}$ is $70.23\left(\mathrm{~g} / \mathrm{cm}^{3}\right)^{-1}$ with $8.63 \%$ standard error. The absolute error for the calculated NPT slope, taking the experimental results as reference, is $16.67 \%$, while for the CPMD it is $46.56 \%$. A bigger set would yield better precision and confidence to the statistical measures, but it is out of the scope of this work.
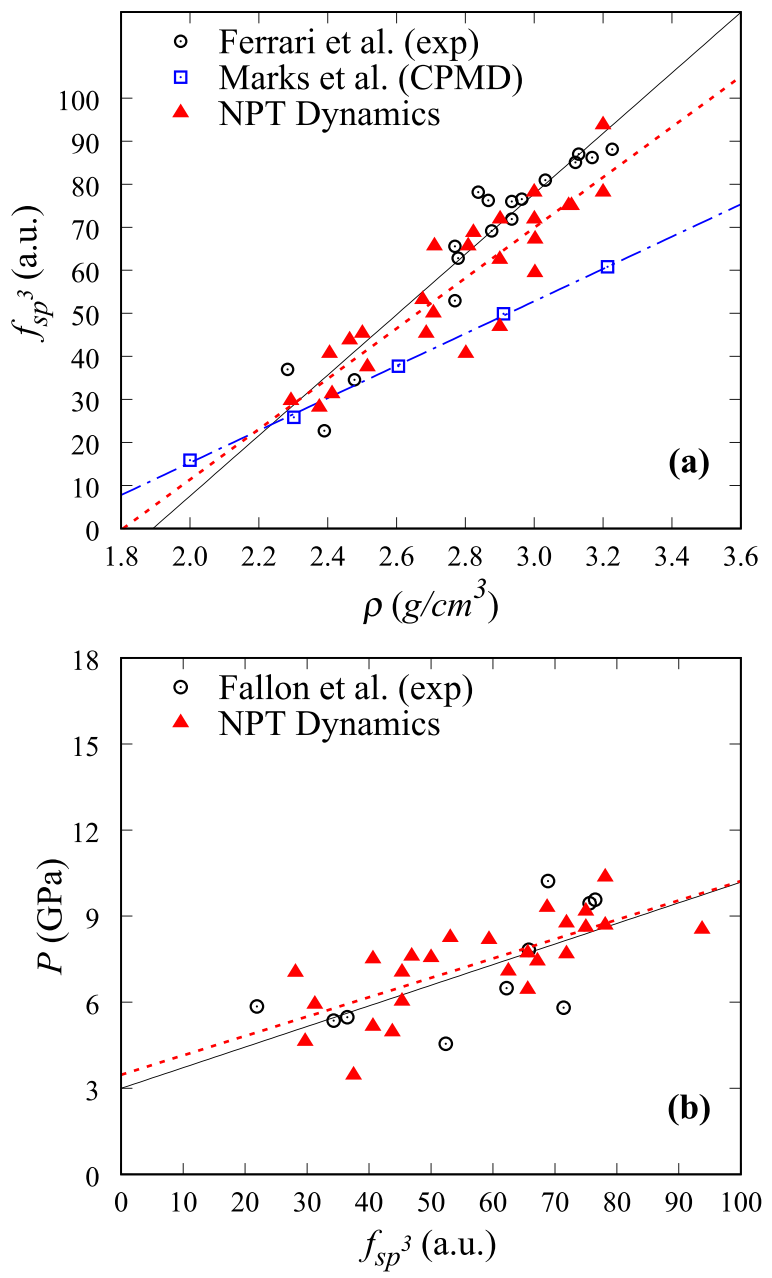

FIG. 4. (a) Calculated $s p^{3}$ fraction as a function of the sample density (red dotted line). For comparison, we include experimental results from Fallon et al. ${ }^{40}$ (black solid line), and computational CPMD results from Marks et al. ${ }^{16}$ (blue dashed line). (b) Pressure as a function of the $s p^{3}$ fraction (red dotted line). We also include experimental results from Fallon et al. ${ }^{40}$ (black solid line).
Figure 4(b) shows the pressure as a function of the $s p^{3}$ fraction for the same points in Fig. 4(a). The error of the fitted slope with respect to experiment is $6.03 \%$. Samples with a pressure closer to the experimental values, accurately predict $s p^{3}$ fraction as a function of $\rho$, and are therefore, used here for the electronic structure analysis and electron transport calculations.

\section{B. Bulk a-C electronic structure}

Amorphous semiconductors have a pseudogap, i.e., there are midgap states (traps) around the Fermi level. These are $\pi$ states that lie between the $\sigma$ gap $^{13}$ and are localized due to the random potential around them (Anderson localization). ${ }^{51}$ The degree of electronic states' localization can be determined using a participation ratio analysis. This method was introduced by Tritsaris et al. to the analysis of a-C structures,${ }^{18}$ and it has the advantage of using the PDOS on each atom, instead of the single electron wave functions, which facilitates the localization calculation. ${ }^{52}$ The degree of localization $w(E)$ can be defined as follows:

$$
w(E)=\sum_{\alpha=1}^{N} \frac{\left(N \times g_{\alpha}(E) / g^{\prime}(E)-1\right)^{2}}{N(N-1)} .
$$

Here $g_{\alpha}(E)=\sum_{i=2 s, 2 p} g_{\alpha, i}$ is the PDOS on site $\alpha, g^{\prime}(E)$ $=\sum_{\alpha} g_{\alpha}(E)$ is the sum of all PDOS, and $N$ is the number of atoms in the system $(N=64$ atoms). For an extended state $g_{\alpha}(E) \rightarrow g^{\prime}(E) / N$ and $w(E) \rightarrow 0$, while for a state strictly localized on atom $\alpha, g_{\alpha}(E)=g^{\prime}(E)$ and $w(E)=1$.

In Fig. 5, we show the DOS and $w(E)$ for two of the samples with low $\left(2.29 \mathrm{~g} / \mathrm{cm}^{3}\right)$ and high $\left(3.2 \mathrm{~g} / \mathrm{cm}^{3}\right)$ densities. We see that $w(E)$ increases with density, as others reported in related work. ${ }^{19}$ For the low-density sample, a continuum of states exists around $E_{F}$ delimited by two gaps around -1.11 and $1.86 \mathrm{eV}$ (averaged from three samples, all with $\rho=2.3 \mathrm{~g} / \mathrm{cm}^{3}$, approximately), while for the highdensity sample, states around $E_{F}$ are distinguishable from each other and the gaps are located around -1.91 and $1.80 \mathrm{eV}$ (averaged from two samples with $\rho=3.20 \mathrm{~g} / \mathrm{cm}^{3}$ ). There is also a small gap between the states around $E_{F}$ that opens with density, but its particular value for each sample is distributed statistically, as the presence of the gap is not only determined exclusively by the amount of $s p^{3}$, but also by the spatial correlation between $s p^{2}$ sites. ${ }^{13}$ This behavior is characteristic of a pseudogap where localized "defect" states are present within the optical gap. ${ }^{14,53}$

Contributions due to $s p^{3}$ and $s p^{2}$ sites to the DOS and $w(E)$ are shown in Fig. 6 for a $3.20 \mathrm{~g} / \mathrm{cm}^{3}$ a-C sample. Most of the localized electronic states are contributed by the $s p^{2}$ sites, while the localization due to $s p^{3}$ sites is located mainly at the conduction band's edge, in good agreement with previous work. ${ }^{14}$ The PDOS for $s p^{2}$ sites is higher than for $s p^{3}$ sites within the pseudogap, which confirms that the localized electronic states that determine conduction are $s p^{2}$-like. Because the $s p^{2} / s p^{3}$ fraction is directly related to the density, the latter will determine the transport mechanism of a-C.

Keeping in mind that $\pi$ states around $E_{F}$, contributed by $s p^{2}$ sites, ${ }^{13}$ will determine the transport mechanism for low 

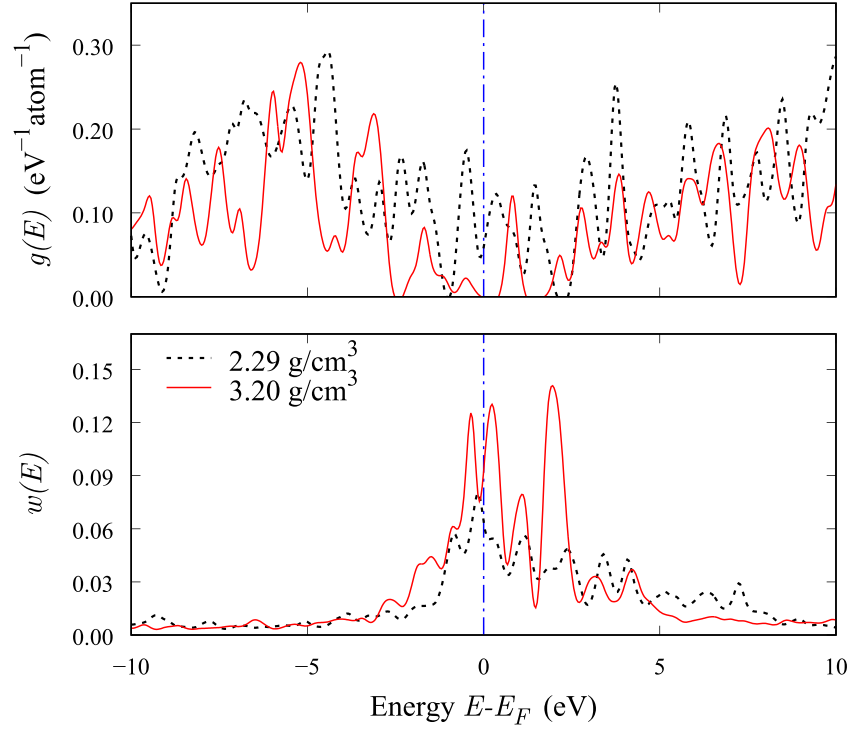

FIG. 5. The top panel shows the total DOS and the bottom panel shows the degree of localization $w(E)$ of a-C samples with densities 2.29 (black dotted line) and $3.20 \mathrm{~g} / \mathrm{cm}^{3}$ (red solid line). The Fermi level's position is marked with a blue dashed line.

bias, and that these are highly localized, we fitted a weighted Lorentzian function to the $w(E)$ points within an energy window between -1.5 and $1.5 \mathrm{eV}$ around $E_{F}$, in order to quantify localization parameters that help us determine different transport regimes as a function $\rho$ and $s p^{3}$ (see Fig. 7)

$$
w_{f i t}(E)=A \frac{\Gamma}{\left(E-\varepsilon_{0}\right)^{2}+\Gamma^{2}} .
$$

Here, $A$ is a fitting constant proportional to the peak magnitude of $w(E), \Gamma$ is a parameter, which determines the width over the

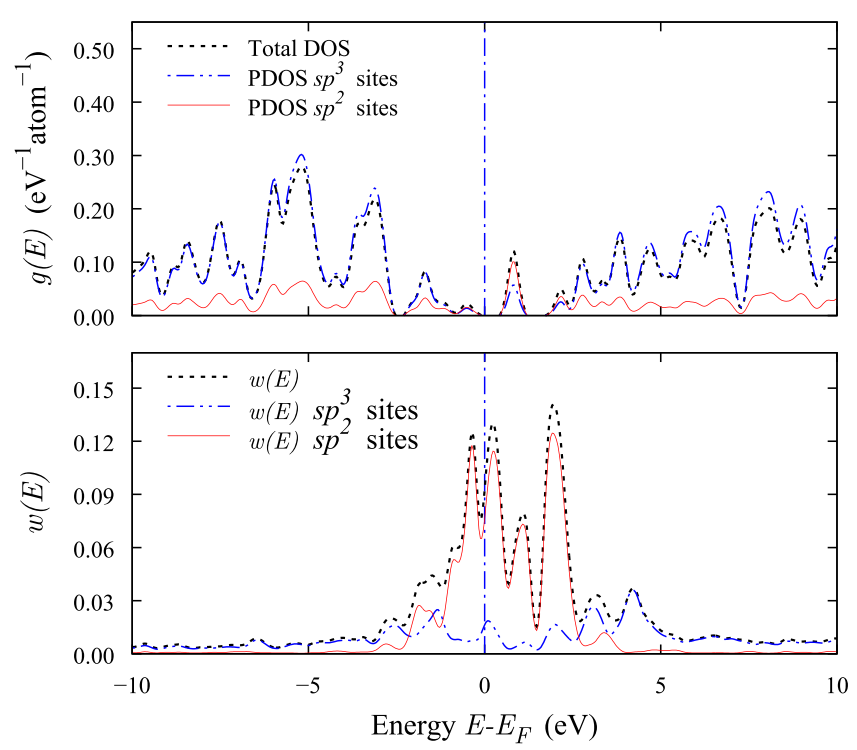

FIG. 6. PDOS (top) and degree of localization (bottom) of an a-C sample of density $3.20 \mathrm{~g} / \mathrm{cm}^{3}$. The sum of the contributions of $s p^{2}$ and $s p^{3}$ sites, obtained with the PDOS on the individual atoms, are represented as red solid and blue dashed lines, respectively. The total DOS and degree of localization are plotted with black dotted lines. The midgap localized states are contributed mostly by $s p^{2}$ sites, while localized $s p^{3}$ sites are located mainly at the band edges energy, and $\varepsilon_{0}$ determines the position of the peak, relative to the Fermi level. The magnitude of the peak of $w_{f i t}(E)$, and its value at the Fermi level can then be calculated as $w_{f i t}\left(\varepsilon_{0}\right)$ $=A / \Gamma$ and $w_{f i t}\left(E_{F}\right)=A \Gamma /\left(\varepsilon^{2}+\Gamma^{2}\right)$ correspondingly.

In Fig. 8, we plotted both the $w_{\text {fit }}\left(\varepsilon_{0}\right)$ and $w_{\text {fit }}\left(E_{F}\right)$ as a function of the density and $f_{s p^{3}}$. Note that $w_{f i t}\left(\varepsilon_{0}\right) \approx w_{f i t}\left(E_{F}\right)$, which indicates that the $s p^{2}$ localized states' energies lie around $E_{F}$. The deviations from $E_{F}$ can be explained by the fluctuations of our calculated points from the experimental $\rho$ vs. $f_{s p^{3}}$ curve. These are significantly higher for densities around $2.8 \mathrm{~g} / \mathrm{cm}^{3}$, as Fig. 4(a) shows.

We approximated the trend of the data points to a Bezier polynomial. Three different trends can be differentiated with respect to the density and $f_{s p^{3}}$ : (1) for low densities $\left(\rho \leq 2.55 \mathrm{~g} / \mathrm{cm}^{3}\right)$ and $f_{s p^{3}} \leq 44.94$, the localization increases linearly; (2) for middle-range densities $\left(2.55 \mathrm{~g} / \mathrm{cm}^{3} \leq \rho \leq\right.$ $\left.2.80 \mathrm{~g} / \mathrm{cm}^{3}\right)$ and $\left(44.94 \leq f_{s p^{3}} \leq 59.64\right)$, the localization is constant; (3) for high densities $\left(\rho \geq 2.80 \mathrm{~g} / \mathrm{cm}^{3}\right)$ and $f_{s p^{3}} \geq$ 59.64 , the localization increments exponentially with respect to the density and $f_{s p^{3}}$. Essentially, we can expect these three regions, where the Fermi localization behaves differently to have different transport mechanisms. Next, we show how this is indeed the case.

\section{Electron transport in a-C films}

After contacting with the electrodes, the system loses periodicity in the transport direction $(z)$. Furthermore, the relaxation at the interfaces with the metallic electrodes changes the atoms' positions and their bonding nature, becoming more $s p^{2}$, similar to what happens at a-C surfaces. ${ }^{19,20}$ The variation in the atoms' positions changes the electronic structure of the sample, see Fig. 9. The new contacted system loses the electronic states gap for both high- and low-density samples and the electronic states' localization (calculated using the PDOS [see Equations (4) and (9)] on the carbon atoms within the scattering region) drops. The effect is stronger in samples with higher densities, in which localized $s p^{2}$ states are very confined in space due to the potential of dominant $s p^{3}$ sites. Confinement is lost when the interfaces reconfigure. We have shown that the high-density systems are shorter (in the $z$ direction) than the low-density ones for a constant number of atoms. In the case of the highest density studied $\left(3.2 \mathrm{~g} / \mathrm{cm}^{3}\right)$, the length of the unit cell in the transport direction is $13.83 \AA$, because we used $5 \AA$ a-C slabs on each

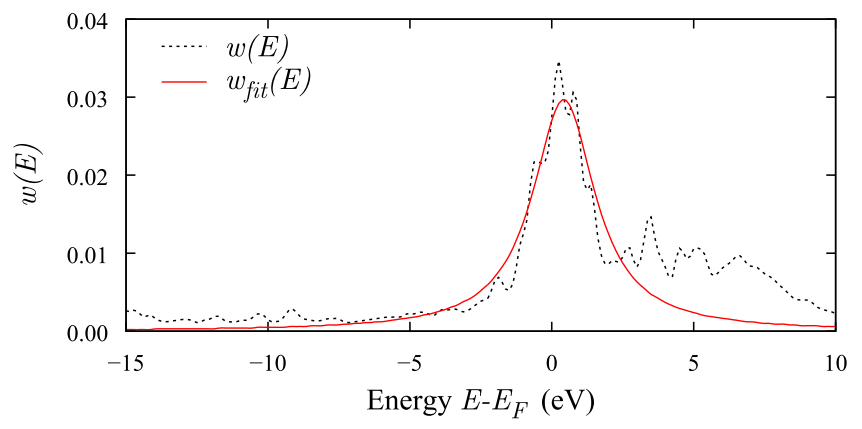

FIG. 7. Degree of localization $w(E)$ and fitted Lorentzian function $w_{f i t}(E)$ of a $2.29 \mathrm{~g} / \mathrm{cm}^{3}$ sample. The fitted function's peak and width are useful to analyze how the localization depends on the density. 

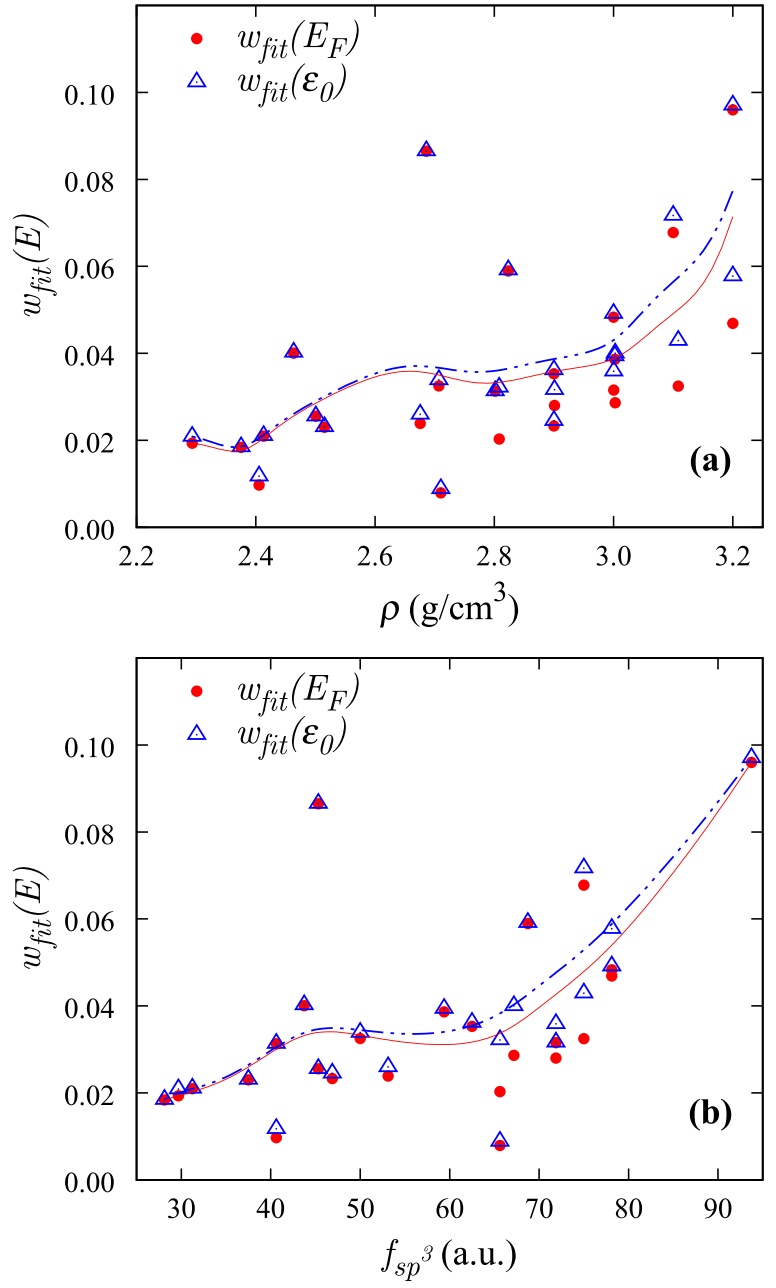

FIG. 8. (a) Localization as a function of the density, and (b) localization as a function of the $s p^{3}$ fraction $f_{s p^{3}}$. The blue triangles are the peak values of the fitted localization $w_{f i t}\left(\varepsilon_{0}\right)$ and the blue dashed line is the fitted Bezier polynomial. The red circles are the value of the fitted localization at the Fermi level $w_{f i t}\left(E_{F}\right)$ and the red solid line is the fitted Bezier polynomial. The localized states are located around $E_{F}$ and three regimes of localization with $\rho$ and $f_{s p^{3}}$ can be distinguished.

side to build the interfaces, from which only $2 \AA$ were fixed, $43 \%$ of the original bulk structure is free to reconfigure. This percentage, explains the drop on the localization peak, stronger for high density than for low-density samples.

Figure 10 shows the electron transmission function together with the PDOS on the $s p^{2}$ and $s p^{3}$ sites, providing qualitative information on the contribution of each hybridization to the available electronic states in the scattering region. PDOS has been widely used in the literature to analyze the transport properties of molecular junctions and nanoscale systems. ${ }^{21-24}$ However, the strategy of PDOS for transport analysis is subject to debate. PDOS changes depending on the chosen basis set and the subspace on which the Hamiltonian is diagonalized. ${ }^{54}$ This is a challenge for the analysis of transport from the electronic structure.

We see that the shape of the transmission function follows the shape of the $s p^{3}$ PDOS for both low- and highdensity samples, since it determines the pseudogap. However, the transmission function of the low-density sample contains resonances around the Fermi level, which are

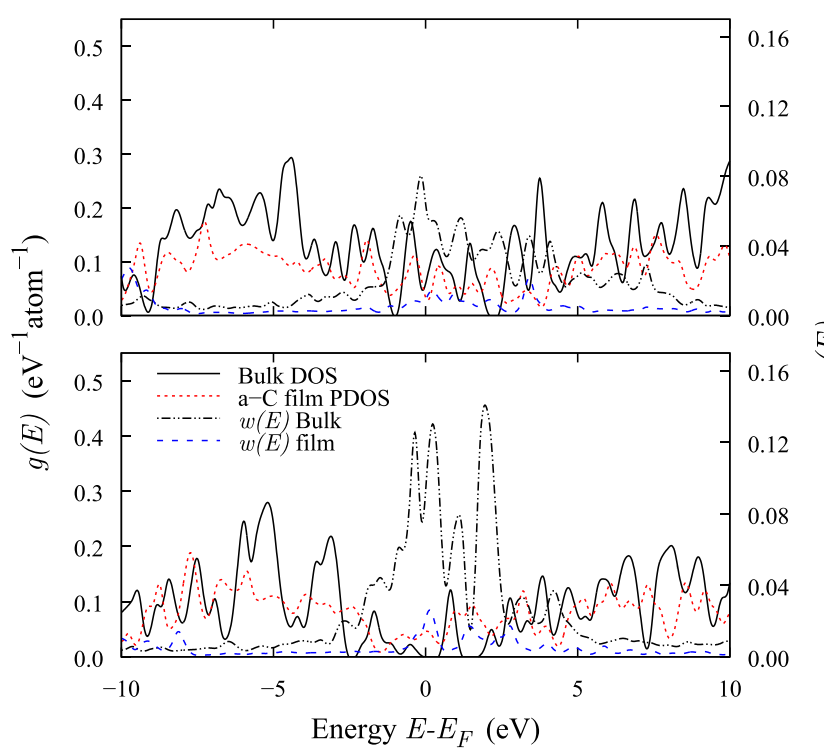

FIG. 9. Bulk a-C DOS (black solid line), and the degree of localization $w(E)$ (black dashed line) on the secondary right axis. After contact with Au electrodes, the a-C thin film is created. Thin-film PDOS on $\mathrm{C}$ atoms (red dotted line) and the degree of localization (red dashed line) are also plotted. We show two a-C samples with $\rho=2.29 \mathrm{~g} / \mathrm{cm}^{3}$ (top) and $\rho=3.2 \mathrm{~g} / \mathrm{cm}^{3}$ (bottom), where the effect of dimensionality and contacts on the localization of high-density samples is notorious.

neither present in the $s p^{2}$ or the $s p^{3}$ PDOS. Resonances in the transmission function are usually associated to highly localized or quasi-bound (QB) states in the transport path. Such localized states lead to RT, which can be measured by changes in the $I-V$ curve's slope and/or negative differential resistance. ${ }^{55}$

RT transport is characteristic of composite materials and semiconductor heterostructures; it has been studied

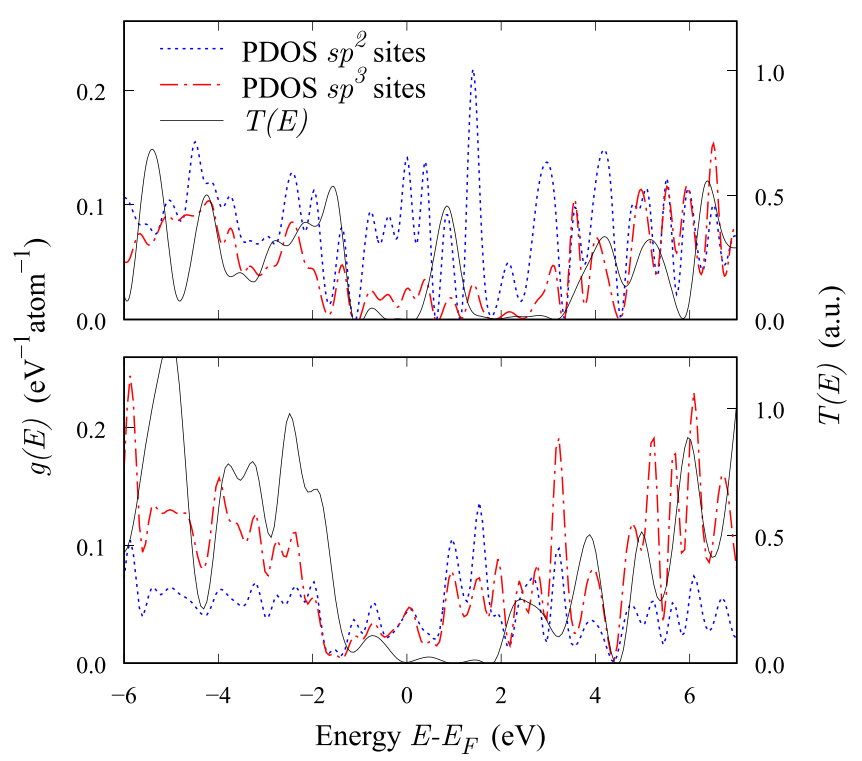

FIG. 10. PDOS on $s p^{2}$ and $s p^{3}$ sites (blue dotted and red dashed lines, respectively), and the transmission function (black solid line) on the secondary right axis. Two a-C samples with $\rho=2.46 \mathrm{~g} / \mathrm{cm}^{3}$ (top panel) and $\rho=$ $3.20 \mathrm{~g} / \mathrm{cm}^{3}$ (bottom panel) are shown. The low-density sample has a resonance around $E_{F}$, indicating that there are localized states at the same or close in energy, while the high-density sample shows a transmission gap around the Fermi level, indicating low electron density. 
experimentally and theoretically. ${ }^{1,56-60}$ RT transport is calculated from the system's potential profile, which lets us calculate the localized states' energies. We divided the system into six slices along the transport direction and determined the available states and localization from the PDOS and $w(E)$ for each individual slice. We also calculated the density distribution along the transport directions and the contributions to the density due to $s p^{2}$ and $s p^{3}$ sites: $\rho_{s p^{2}}$ and $\rho_{s p^{3}}$, respectively. To calculate $\rho, \rho_{s p^{2}}$, and $\rho_{s p^{3}}$ along the transport direction, we divided the system here into 50 slices. The points were smoothed using a Bezier curve with the calculated points as the polynomial parameters. In Fig. 11 we plotted $w(E)$ and the density distribution for the same samples used in Fig. 10. These results show that the localization is directly related to the $s p^{3}$ distribution within the system. For the longer, low-density sample, a region exists between $10 \AA$ and $12 \AA$ where the $s p^{3}$ content rises, creating a potential barrier and causing the $s p^{2}$ electronic states to get localized, around 7 and $15 \AA$. We have only considered the features of $\rho$ and $w(E)$ in one dimension, but it is worth mentioning that clusters of $s p^{2}$ sites might be distributed in 3D, influencing the transport mechanism. From Fig. 11(a) we see the similarity of the a-C with a square quantum well, which was the model proposed by Katkov and Bhattacharyya. ${ }^{8}$ However, their model considered symmetric wells with sharp barriers in the electrostatic potential (associated with $s p^{3}$ clusters), which is not the case for realistic disordered structures as the ones we present. The influence of this geometric asymmetries can be seen in the difference in the energy position of the QB states, their energy width, and localization degree. Although the localized states at each side of the barrier are not at the same energy (due to the wells' asymmetry), there is a considerable tail overlapping that is responsible for the resonance in $T(E)$, thus, evidencing RT transport as reported by Bhattacharyya et al. ${ }^{1,12}$

Our results can also be contrasted with the experimental results from Protopopova et al. ${ }^{61}$ They studied the thickness dependence of the conductivity in a-C ultra-thin films, and proposed that for thinner films $(\leq 4 \mathrm{~nm})$ the conduction decreases due to the filling of exponentially distributed traps. On the contrary, we propose that conduction in high-density

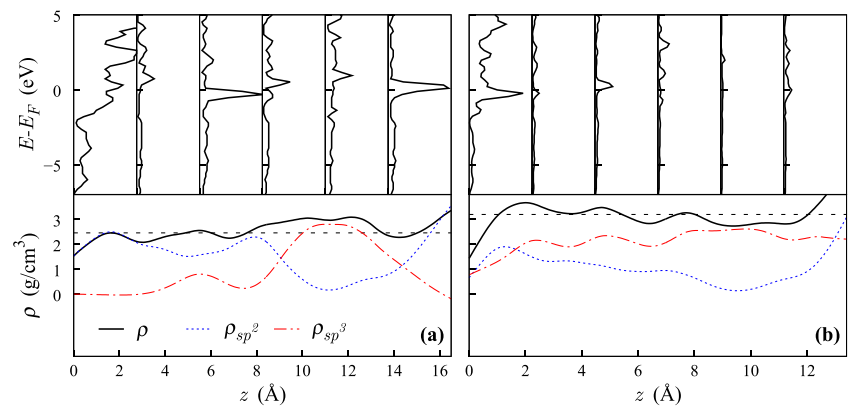

FIG. 11. The top panel shows the degree of localization $w(E)$ in different spatial regions along the transport direction $z$. The bottom panel shows the density $\rho$ (black solid line), and the $s p^{2}$ and $s p^{3}$ contributions to the total density $\rho_{s p^{2}}$ (blue dotted) and $\rho_{s p^{3}}$ (red dashed), respectively, averaged in the $x y$ plane along the transport direction $z$. The system's average density is shown as a horizontal black dashed line for reference. Two systems are shown: (a) $\rho=2.46 \mathrm{~g} / \mathrm{cm}^{3}$ and (b) $\rho=3.20 \mathrm{~g} / \mathrm{cm}^{3}$. ultra-thin films decreases due to the reduction in the number of charge traps (localized electronic states) induced by the atomic reconfiguration.

Computing $T(E)$ self-consistently using the DFT-NEGF method for each of the samples is a computationally expensive task. Moreover, to calculate the $I-V$ curves with Equation (8), one has to compute $T(E)$ at each bias point. Therefore, we first calculated $I-V$ characteristic curves using a reduced single- $\zeta$ polarized (SZP) basis set, as shown in Fig. 12. As the density of the sample increases, there is a change in the shape of the $I-V$ characteristic curve. The $I-V$ curve in Fig. 12(a) is similar to a linear response with minor slope changes. We attribute these changes to weakly localized states, but the dominant transport mechanism is semimetallic through extended $s p^{2} \pi-\pi^{*}$ states. On the other hand, in Fig. 12(b) we see notorious changes in the slope of the $I-V$ curves, and for the case of the $2.67 \mathrm{~g} / \mathrm{cm}^{3}$ sample, negative differential resistance around 1.3 and $1.7 \mathrm{~V}$. This is evidence of RT and variable range hopping (VRH) transport, induced by strongly localized $s p^{2}$ states around $E_{F}$. Finally, in Fig. 12(c), we see linear characteristics for low biases. The slope changes as the voltage increases, which is a common feature in systems whose dominant transport mechanism is thermally activated. ${ }^{27,62}$

These results are only qualitative, since a SZP basis set is unable to represent accurately the energetics of a-C, especially in high-density samples, as shown in previous works. ${ }^{32,33}$ Therefore, we calculated the $I-V$ curves for a set of samples with densities ranging from 2.23 to $3.20 \mathrm{~g} / \mathrm{cm}^{3}$, using a DZP basis set, as shown in Fig. 13. The $I-V$ curve for the sample with $\rho=2.46 \mathrm{~g} / \mathrm{cm}^{3}$, shows fluctuations in the slope, characteristic of RT transport, which is consistent with the localized states observed in Fig. 11(a), while the $I-V$ characteristic for the sample with $\rho=3.20 \mathrm{~g} / \mathrm{cm}^{3}$ is linear, for activated transport and consistent with the results in Fig. 13(b).
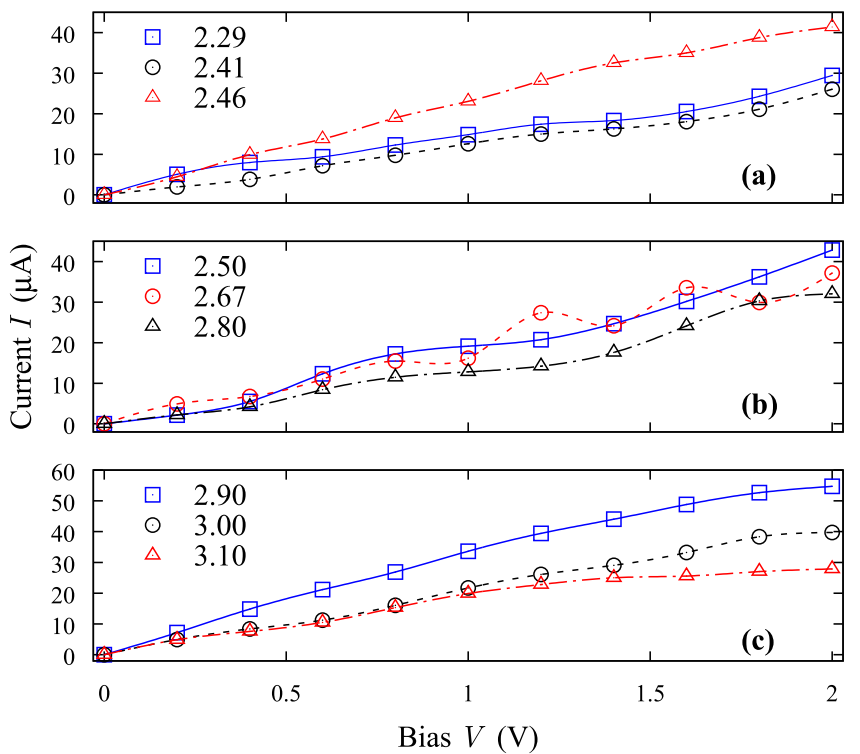

FIG. 12. Current-voltage $(I-V)$ characteristic curves of a-C thin films with different densities, calculated using DFT-NEGF self-consistent method and a SZP basis set. We identify three types of conduction: (a) semimetallic for the low-density samples, (b) RT and VRH for middle-range densities and (c) activated for the high-density samples. 
These transitions from one conduction mechanism to the other have been observed previously by Chimowa, Churochkin, and Bhattacharyya ${ }^{63}$ for boron-doped nano-crystalline films. They showed that the transitions are heavily dependent on Nitrogen concentrations at low temperatures. However, the dominant conduction mechanism is also dependent on the temperature. They found that for concentrations of $10 \%$ to $15 \%$ Nitrogen, at temperatures below $118 \mathrm{~K}$, the dominant conduction mechanism is VRH, while for concentrations of $20 \%$ Nitrogen, activated conduction was evidenced in the range of $28 \mathrm{~K}$ to $89 \mathrm{~K}$. These conclusions are applicable to our findings, since the role of Nitrogen doping is to increase the level of disorder, i.e., to increase the fraction of $s p^{2}$ sites in the film. A more rigorous computational study of the temperature dependence of these transitions is needed to establish the range of temperatures in which our model is valid.

\section{CONCLUSIONS AND FUTURE WORK}

Using an algorithm based on the underlying physical process modeled with NPT ensemble dynamics, we generated 25 bulk a-C samples, which were used to build ultrathin film systems. This allowed us to study the transport's evolution, the localization as function of the $s p^{3}$ content, and density. We used Kohn-Sham DFT and the NEGF formalism to calculate the electron transmission and transport mechanisms. We identified three transport regimes, depending on the density. For very low densities $\left(\rho \leq 2.4 \mathrm{~g} / \mathrm{cm}^{3}\right)$, the dominant transport mechanism is semimetallic. In high-density systems $\left(\rho \geq 2.9 \mathrm{~g} / \mathrm{cm}^{3}\right)$, transport is driven by variable range and thermally activated hopping. For middle-range densities $\left(2.4 \mathrm{~g} / \mathrm{cm}^{3} \leq \rho \leq 2.9 \mathrm{~g} / \mathrm{cm}^{3}\right)$, RT and hopping (induced due to the appearance of localized states) dominate.

We demonstrated that contact with electrodes drops the localization of ultra-thin films' electronic states, affecting the electron transport mechanisms. The contacts' effect is

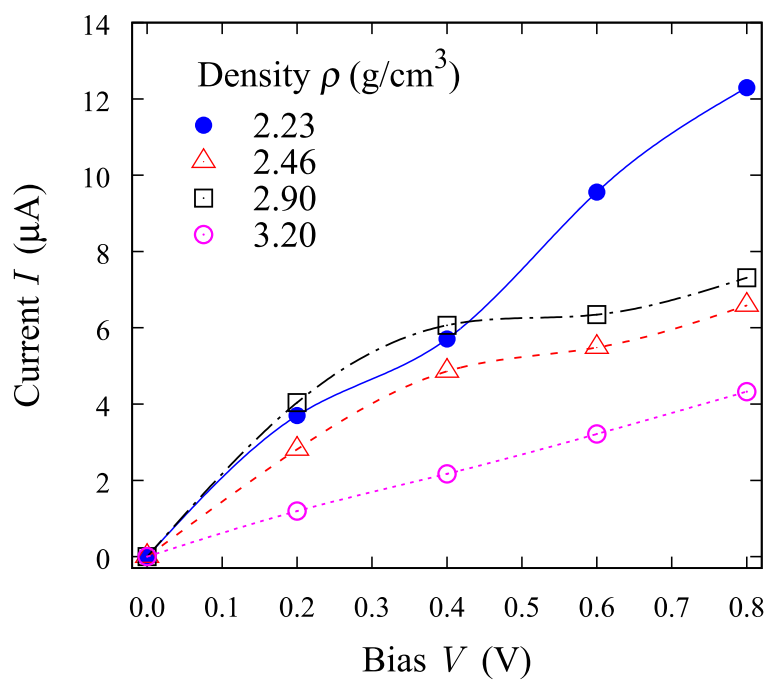

FIG. 13. $(I-V)$ curves calculated using a high-quality DZP basis set. The three types of conduction can be seen: (1) semimetallic for the low-density sample $\rho=2.29 \mathrm{~g} / \mathrm{cm}^{3}$ (blue filled circles); (2) hopping and resonance tunneling for middle-range densities $\rho=2.46$ and $2.90 \mathrm{~g} / \mathrm{cm}^{3}$ (red triangles and black squares); and (3) activated for the high-density sample $\rho=3.20 \mathrm{~g} /$ $\mathrm{cm}^{3}$ (magenta open circles). especially strong for high-density films, and our findings are in agreement with experimental works. These findings can impact materials engineering significantly, providing essential information for designing future sensors and devices envisioned by the electronics industry.

While we were able to differentiate between density regions, we have yet to establish sharp thresholds. This requires further calculations and adopting more sophisticated techniques to obtain a quantitative model. We must also take into account electron-phonon interactions and phase-incoherent processes (which have not been considered), to improve the description of electron transport in aC films. A different approach needs to be taken, either by considering a mean-field approach, similar to the recent studies by McIntosh et al.$^{64}$ on superconductivity in borondoped diamond, or by using the DFT-NEGF method, addressing electron-phonon interaction by means of perturbation theory at the level of self-consistent Born approximation. ${ }^{46}$

\section{SUPPLEMENTARY MATERIAL}

See supplementary material for a more detailed view of the pseudogap and electronic gap and for more $I-V$ characteristic curves of individual samples.

\section{ACKNOWLEDGMENTS}

We are grateful to Miguel Caro from the Department of Applied Physics at Aalto University for his helpful discussion on amorphous carbon generation and analysis of the electronic structures in bulk samples, and to Claudia Gomes da Rocha from Trinity College, Dublin for her insightful comments during the manuscript's preparation and valuable discussions on transport calculations. The calculations were performed on the Triton Cluster (funded by the Science-IT project at Aalto University), the Taito Cluster of the Finnish CSC-IT Center for Science, and the HPC Cluster of the Vicerrectoría de Investigaciones at Los Andes University. We also acknowledge support from Los Andes University and Universidad del Valle (through a shared grant for research between the Engineering faculties), and the Academy of Finland (Project Nos. 279240 and 251748).

${ }^{1}$ S. Bhattacharyya, S. J. Henley, E. Mendoza, L. Gomez-Rojas, J. Allam, and S. R. P. Silva, Nat. Mater. 5, 19 (2006).

${ }^{2}$ R. U. R. Sagar, X. Zhang, C. Xiong, and Y. Yu, Carbon N.Y. 76, 64 (2014).

${ }^{3}$ T. Laurila, A. Rautiainen, S. Sintonen, H. Jiang, E. Kaivosoja, and J. Koskinen, Mater. Sci. Eng. C 34, 446 (2014).

${ }^{4}$ T. Laurila, V. Protopopova, S. Rhode, S. Sainio, T. Palomäki, M. Moram, J. M. Feliu, and J. Koskinen, Diamond Relat. Mater. 49, 62 (2014).

${ }^{5}$ D. Luo, H. Xu, M. Zhao, M. Li, M. Xu, J. Zou, H. Tao, L. Wang, and J. Peng, ACS Appl. Mater. Interfaces 7, 3633 (2015).

${ }^{6}$ International Technology Roadmap for Semiconductors (ITRS), 1, 2013.

${ }^{7}$ S. Bhattacharyya and S. Silva, Thin Solid Films 482, 94 (2005).

${ }^{8}$ M. V. Katkov and S. Bhattacharyya, J. Appl. Phys. 111, 123711 (2012).

${ }^{9}$ M. V. Katkov, R. McIntosh, and S. Bhattacharyya, J. Appl. Phys. 113, 093701 (2013).

${ }^{10}$ M. V. Katkov and S. Bhattacharyya, J. Appl. Phys. 113, 183712 (2013).

${ }^{11}$ S. R. P. Silva, G. A. J. Amaratunga, C. N. Woodburn, M. E. Welland, and

S. Haq, Jpn. J. Appl. Phys., Part 1 33, 6458 (1994).

${ }^{12}$ S. Bhattacharyya, Appl. Phys. Lett. 91, 142116 (2007).

${ }^{13}$ J. Robertson and E. O'Reilly, Phys. Rev. B 35, 2946 (1987). 
${ }^{14}$ D. A. Drabold, P. A. Fedders, and P. Stumm, Phys. Rev. B 49, 16415 (1994).

${ }^{15}$ N. A. Marks, in Computer-Based Modeling of Novel Carbon Systems and Their Properties, edited by L. Colombo and A. Fasolino (Springer, 2010), Vol. 3, Chap. 5, pp. 129-170.

${ }^{16}$ N. A. Marks, D. R. McKenzie, B. A. Pailthorpe, M. Bernasconi, and M. Parrinello, Phys. Rev. B 54, 9703 (1996).

${ }^{17}$ N. A. Marks, N. C. Cooper, D. R. McKenzie, D. G. McCulloch, P. Bath, and S. P. Russo, Phys. Rev. B 65, 075411 (2002).

${ }^{18}$ G. A. Tritsaris, C. Mathioudakis, P. C. Kelires, and E. Kaxiras, J. Appl. Phys. 112, 103503 (2012)

${ }^{19}$ M. A. Caro, R. Zoubkoff, O. Lopez-Acevedo, and T. Laurila, Carbon N.Y. 77, 1168 (2014)

${ }^{20}$ M. A. Caro, J. Määttä, O. Lopez-acevedo, and T. Laurila, J. Appl. Phys. 117, 034502 (2015).

${ }^{21}$ S. Caliskan and A. Laref, Sci. Rep. 4, 7363 (2014).

${ }^{22}$ R. Gutiérrez, F. Grossmann, and R. Schmidt, ChemPhysChem 4, 1252 (2003).

${ }^{23}$ A. Sen, C. J. Lin, and C. C. Kaun, J. Phys. Chem. C 117, 13676 (2013).

${ }^{24}$ X. Q. Shi, Z. X. Dai, X. H. Zheng, and Z. Zeng, J. Phys. Chem. B 110, 16902 (2006).

${ }^{25}$ N. F. Mott, Philos. Mag. 19(160), 835-852 (1969).

${ }^{26}$ Q. Z. Xue and X. Zhang, Carbon N.Y. 43, 760 (2005).

${ }^{27}$ A. Tibrewala, E. Peiner, R. Bandorf, S. Biehl, and H. Lüthje, Appl. Surf. Sci. 252, 5387 (2006).

${ }^{28}$ P. Hohenberg and W. Kohn, Phys. Rev. 136, B864 (1964).

${ }^{29}$ J. P. Perdew, K. Burke, and Y. Wang, Phys. Rev. B 54, 16533 (1996).

${ }^{30}$ J. J. Mortensen, L. B. Hansen, and K. W. Jacobsen, Phys. Rev. B 71, 035109 (2005).

${ }^{31}$ P. E. Blöchl, Phys. Rev. B 50, 17953 (1994).

${ }^{32}$ J. S. Nelson, E. B. Stechel, A. F. Wright, S. J. Plimpton, P. A. Schultz, and M. P. Sears, Phys. Rev. B 52, 9354 (1995).

${ }^{33}$ P. A. Schultz and E. B. Stechel, Phys. Rev. B 57, 3295 (1998).

${ }^{34}$ H. J. Monkhorst and J. D. Pack, Phys. Rev. B 13, 5188 (1976).

${ }^{35}$ C. Broyden, IMA J. Math. Appl. 6, 76 (1970).

${ }^{36}$ R. Fletcher, Comput. J. 13, 317 (1970).

${ }^{37}$ D. Goldfarb, Math. Comput. 24, 23 (1970).

${ }^{38}$ D. F. Shanno, Math. Comput. 24, 647 (1970).

${ }^{39}$ H. J. C. Berendsen, J. P. M. Postma, W. F. van Gunsteren, A. DiNola, and J. R. Haak, J. Chem. Phys. 81, 3684 (1984).

${ }^{40}$ P. J. Fallon, V. S. Veerasamy, C. A. Davis, J. Robertson, G. A. J. Amaratunga, W. I. Milne, and J. Koskinen, Phys. Rev. B 48, 4777 (1993).

${ }^{41}$ A. C. Ferrari, A. Libassi, B. K. Tanner, V. Stolojan, J. Yuan, L. M. Brown, S. E. Rodil, B. Kleinsorge, and J. Robertson, Phys. Rev. B 62, 11089 (2000).
${ }^{42}$ D. G. McCulloch, D. R. McKenzie, and C. M. Goringe, Phys. Rev. B 61, 2349 (2000)

${ }^{43}$ J. Taylor, H. Guo, and J. Wang, Phys. Rev. B 63, 245407 (2001).

${ }^{44}$ Y. Xue, S. Datta, and M. A. Ratner, Chem. Phys. 281, 151 (2002).

${ }^{45}$ M. Brandbyge, J.-L. Mozos, P. Ordejón, J. Taylor, and K. Stokbro, Phys. Rev. B 65, 165401 (2002).

${ }^{46}$ T. Frederiksen, M. Paulsson, M. Brandbyge, and A.-P. Jauho, Phys. Rev. B 75, 205413 (2007).

${ }^{47}$ J. Chen, K. S. Thygesen, and K. W. Jacobsen, Phys. Rev. B 85, 155140 (2012).

${ }^{48}$ L. Bengtsson, Phys. Rev. B 59, 12301 (1999).

${ }^{49}$ S. Datta, Cambridge Studies in Semiconductor Physics and Microelectronic Engineering (Cambridge University Press, 1995), Vol. 3, p. 377.

${ }^{50}$ S. Datta, Computer Engineering (Cambridge University Press, 2005), p. 419.

${ }^{51}$ P. W. Anderson, Phys. Rev. 109, 1492 (1958).

${ }^{52}$ N. C. Murphy, R. Wortis, and W. A. Atkinson, Phys. Rev. B 83, 184206 (2011).

${ }^{53}$ J. Robertson, Mater. Sci. Eng. R 37, 129 (2002).

${ }^{54}$ T. Rangel, G.-M. Rignanese, and V. Olevano, Beilstein J. Nanotechnol. 6, 1247 (2015).

${ }^{55}$ D. K. Ferry and S. M. Goodnick, Transport in Nanostructures, 2nd ed. (Cambridge University Press, 2009), p. 512.

${ }^{56}$ M. T. Bjoerk, B. J. Ohlsson, T. Sass, A. I. Persson, C. Thelander, M. H. Magnusson, K. Deppert, L. R. Wallenberg, L. Samuelson, M. T. Björk, B. J. Ohlsson, T. Sass, A. I. Persson, C. Thelander, M. H. Magnusson, K. Deppert, L. R. Wallenberg, and L. Samuelson, Appl. Phys. Lett. 80, 1058 (2002).

${ }^{57}$ R. Tsu and L. Esaki, Appl. Phys. Lett. 22, 562 (1973).

${ }^{58}$ M. Cahay, M. McLennan, S. Datta, and M. S. Lundstrom, Appl. Phys. Lett. 50, 612 (1987).

${ }^{59}$ A. P. Horsfield, L. Tong, Y. A. Soh, and P. A. Warburton, J. Appl. Phys. 108, 014511 (2010).

${ }^{60}$ J. F. Rivera, J. C. Arce, and J. Velasco-Medina, IEEE Trans. Nanotechnol. 11, 1174 (2012).

${ }^{61}$ V. Protopopova, A. Iyer, N. Wester, A. Kondrateva, S. Sainio, T. Palomäki, T. Laurila, M. Mishin, and J. Koskinen, Diamond Relat. Mater. 57, 43 (2015).

${ }^{62}$ S. Lampa-Pastirk, J. P. Veazey, K. A. Walsh, G. T. Feliciano, R. J. Steidl, S. H. Tessmer, and G. Reguera, Sci. Rep. 6, 23517 (2016)

${ }^{63}$ G. Chimowa, D. Churochkin, and S. Bhattacharyya, Europhys. Lett. 99, 27004 (2012).

${ }^{64}$ R. McIntosh, N. Mohanta, A. Taraphder, and S. Bhattacharyya, e-print arXiv:1509.09248. 